\title{
Refreshable Braille Displays
}

\author{
B. Bhanushali \\ DJSCE, U-15, \\ J. V.P. D. Scheme \\ Vile Parle $(\mathrm{W})$, Mumbai
}

\author{
Ayush Dhoot \\ DJSCE, U-15, \\ J. V.P. D. Scheme \\ Vile Parle (W), Mumbai
}

\author{
Prachi Gandhi \\ DJSCE, U-15, \\ J.V.P.D.Scheme \\ Vile Parle (W), Mumbai
}

\author{
Kathan Mehta \\ DJSCE, U-15, \\ J. V.P. D. Scheme \\ Vile Parle (W), Mumbai
}

\begin{abstract}
Visual impairment in terms of sight loss and blindness affects 285 million people worldwide. Owing to the recent technological advancements in the fields of electronics and hardware manufacturing, immense progress has taken place towards the availability of e-books for educational and recreational purposes as well as the display devices for the visually impaired. Today, a lot of the devices rely solely on speech output as the means of communication. But when it comes to deep understanding of the information, speech has its limitations especially in subjects like graphics and technical texts. Hence, the blind readers prefer electronic braille displays over speech output devices. But these are prohibitively expensive. Today, the search is on for a low cost, customizable and durable refreshable display device that would help solve the predicament of thousands of visually impaired people worldwide. The aim of this paper is to give a brief overview of the currently available braille printers and braille generators along with a comparative study of their endurance, cost and durability. This paper reviews the braille display technologies that are currently available in the various developed and developing countries and a concept design for an inexpensive and portable refreshable braille generator using electromagnetic relays and solenoids is proposed.
\end{abstract}

\section{Keywords}

Refreshable Braille Display, braille generators, electromagnetic relays, solenoids

\section{INTRODUCTION}

Visual impairment accounts for either a complete loss of, or a reduced ability to perceive light. While modern advancements in optics have ensured that a large portion of minor impairments can now be cured by procedures like laser treatments or even with the help of lenses and spectacles, we are still far off when it comes to dealing with the complications faced by people facing low vision or blindness.

According to a survey conducted by the World Health Organization (WHO) in 2010, around $20 \%$ of the Indian population suffers from either poor sightedness, visual impairment or blindness, with more than 8 million people suffering from permanent blindness. [1] This study clearly shows that the number of people suffering from this impairment cannot be ignored. Due to the lack of vision, the most common problem they face is that of communication with the world. Hence, it is necessary to take serious efforts to make them self-sufficient when it comes to reading and gaining precious information so that they too can become contributing members of society.[2]

The braille system was invented by Louis Braille and since has been the standard language used for blind education. A matrix consisting of $3 \times 2$ raised dots is used in order to classify each of the 26 alphabets, the punctuation and the numbers.[3] The pattern of the raised dots help the person to decode the corresponding message. There are two types of braille, alphabetic braille and literary braille. The former uses a cell to represent one alphabet while the former is also called contracted braille and can fit more than one alphabets in a single cell and thus is used in most books.

\begin{tabular}{|c|c|c|c|c|c|c|c|c|c|}
\hline$\because$ & $\because:$ & $\because$ & $\because$ & $\because$ & $\because$ & $\because$ & $\because$ & $\because$ & $\because:$ \\
\hline a & $b$ & C & $d$ & e & $f$ & $\mathrm{~g}$ & $\mathrm{~h}$ & $\mathrm{i}$ & $\mathrm{j}$ \\
\hline$\because:$ & $\because:$ & $\because$ & $\because$ & $\because$ & $\because:$ & $\because:$ & $\because:$ & & $\because:$ \\
\hline $\mathrm{k}$ & I & $\mathrm{m}$ & $n$ & 0 & $p$ & $q$ & $r$ & $\mathrm{~S}$ & $t$ \\
\hline$\because$ & $\because:$ & $\because:$ & $\because$ & : : & $\because:$ & & & & \\
\hline $\mathrm{u}$ & V & W & $X$ & $y$ & $\mathbf{Z}$ & & & & \\
\hline
\end{tabular}

Fig 1: Braille Alphabet Chart

It can be said without any doubt that today, most blind people are relatively self-sufficient when it comes to being able to read data through the electronic medium, but one question that always persists is whether the means of communication used by the blind is effective and optimal enough to promote deep understanding of the topic. There are two main reasons which prevent braille from becoming the primary communication medium for the blind : 1) Today, the electronic braille readers are costly and range from $\$ 2000$ to $\$ 50,000$ for a large braille pad, and 2) The cost of translating and printing a book in braille is much higher than the normal books. [4] Due to these reasons, the pressure is on us to develop a novel solution for this longstanding issue.

This comparative analysis paper aims to present and contrast the three currently available types of refreshable braille displays: linear, rotating and panda braille and thus to propose an economic and robust prototype which may solve a few of the drawbacks. Finally, some conclusions are drawn.

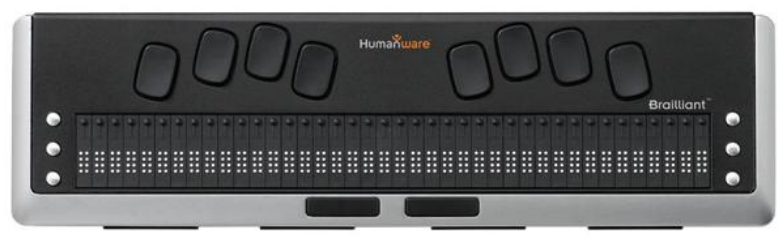

Fig 2: Linear Braille Display

\section{TYPES OF BRAILLE DISPLAYS}

Braille displays or generators used are of refreshable type, that is, once displayed data can be erased and new information can be displayed on the same unit. These Braille generators are used to convert text from a document processed electronically 
into braille characters and present them on display devices in real time.

For braille readers, the most important sensation is the impression of touch which they use to predict the character from the resulting movement of the braille cells. [5]Therefore, it is important for the braille dots to effectively move past finger-tips of the user producing the sensation of indicated characters and enabling the user to create a model of this character in his mind and predict the alphabet or number. For accurate and speedy reading of text, it is pivotal to have number of cells on the display unit. Comparative analysis of linear, rotating and panda refreshable braille displays is studied based on their parameters like mechanism, reliability, complexity and cost.

\subsection{Linear Braille Display}

Linear Refreshable braille displays consists of a single line with number of cells ranging from eight to eighty depending upon the requirement. Each cell is modelled and controlled using actuators which raises the pin in the vertical direction in accordance with the character to be indicated.

Different types of actuators are available in the market, each of them exhibiting peculiar characteristics. [6]Piezo-electric actuators and Solenoids are most commonly used and are the cheapest one amongst all available options. Also, pneumatic actuators, electro-magnetic actuators and shape memory alloys (SMA) are other actuators that can be used in linear refreshable braille displays.Piezo-electric actuators have low power consumption whereas electro-magnetic actuators and shape memory alloys have high power consumption. [7]SMA has a response time of $1 \mathrm{~s}$ and a driving current of $300 \mathrm{~mA}$ which is less as compared to piezoelectric actuators and electromagnetic actuators.

Reading activity is performed by sliding the index smoothly over the cells along the single line from left to right, left indicating the starting cells. As and when ending cells starts getting used, data on the starting cells is erased and can be used to display new information, thus demonstrating refreshable property.

[8]Perkin's braille display was developed for the first time in 1950 and since then it is claimed to be widely used over 170 countries. These braille displays are used by people of all ages enabling the user to implement everyday activities with ease. Another example for linear type is Bristol's braille display, which consists of 4 lines and each line constitutes 28 braille cells. Each cell requires six dots, therefore there are 672 dots in total modelled using 672 actuators. Large number of components make the product bulky and also results in higher wear and tear. Moreover, situations may arise to ship the product back to the country of origin for its repairmen making it difficult to afford to users in developing nations.

\subsection{Rotating Braille Display}

Rotating refreshable Braille display was first developed in USA by National Institute of Standards and Technology (NIST) in the year 1999. This display manifests an infinitely long length of characters along with reduction in the number of actuators used. The number of actuators used are half as compared to the cell dots, that is, for a generator with 8 dots, only 4 actuators are used. This in turn reduces the system size by $50 \%$.

This generator makes use of a disc, and cells are presented only at the circumference with pins representing Braille dots. The retention mechanism of a pin constitutes of two layers, each of them containing three tracks which hold the pin in the intended position as per the desired character. Text is understandable on the top of the wheel with a fraction of wheel exposed as the reading region. The user moves his finger over it and predicts the data as per the sensation produced from those moving pins. The software unit controls the angle with which the wheel is moving.

An example of rotating braille display is Mangold L Type developed by NP Engineering. However, this display makes used of a flexible belt instead of a disc. Furthermore, the unit becomes more compact and also the cost of the entire display reduces. A desirable attribute provided is allowance to set refresh rate. [9]Thus providing more control to the user over its control mechanism.

As the rotating braille display uses a disc instead of an array, the effectiveness of the generator increases as compared to linear display. Moreover, reduction in the number of actuators and greater compactness reduces volume and hence size to a greater extent with a comparable decrement in the cost. However, the mechanism employed for the rotating display is much more complex as compared to that in linear braille displays.

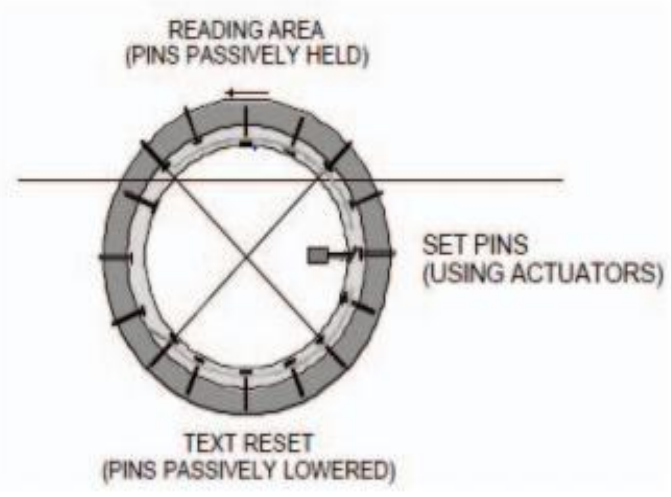

Fig 3: Rotating Braille Display

\subsection{Panda Braille Display}

The Panda Braille Display was developed by the students of VIT University, India. [6]It design structure was based on Electro-cutaneous (EC) stimulations to generate the sensations of dots for corresponding braille characters.

The two main components in Panda Braille display are electrodes and motion sensor. Electrodes represents pins, and motion sensors monitors and manages the speed and location of the braille pins. When fingers come in contact with the electrodes, the circuit is said to be completed and current is produced. The magnitude of the current so produced is enough to generate sufficient sensation required by the user to predict the intended braille character. However, considering the safety measures, proper calibration and protection providing circuits are essential. As there are no moving objects, reduction in wear and tear is achieved which reduces the requirement of maintenance and repairement and as a repairement and as a result of which, considerable amount of decrease in the cost is observed. A few advantages provided by this type of display is its light weight, portability and low power requirement. 


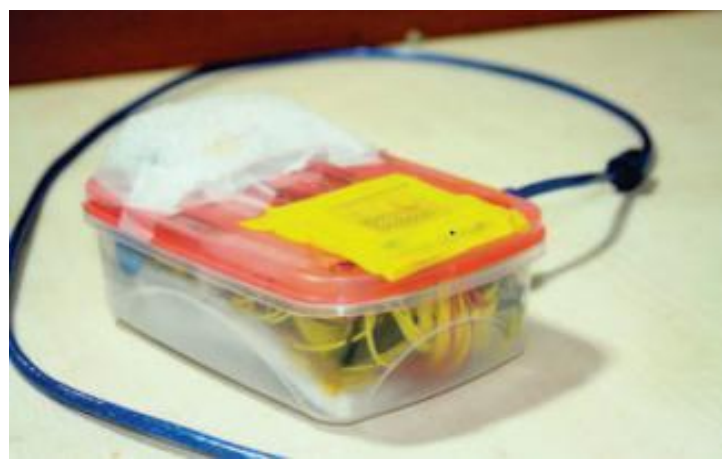

Fig 3: Panda Braille Display

\section{PROPOSED PLAN}

The learning and communication process for the unsighted is a difficult task. The current methods by which the unsighted are able to communicate are few, and all have serious drawbacks. The proposed plan will be effectual in bridging the communication gap between sighted and non-sighted people. The device involves processing of image signals by the technique of Optical Character Recognition. It is possible to use a prevalent image present in the device. Another way of taking the input is downloading the image from the internet. These images are converted to a text file from where the characters are extracted and control signals are generated depending on each character. These control signals drive the Raspberry Pi and speaker accordingly. The control signals produced enable the Raspberry Pi to propel the solenoids of the Braille display. The respective solenoid to be featured experiences thrust from the controller and is pushed upwards. The impetus generated by the six solenoids form different combinations and stand for different Braille letters. The impulses produced by the Braille display allow the blind user to read the Braille alphabet. The second half of the device involves the use of a mobile application. The Braille input of six dots is taken using an Android application. The application consists of six movable dots in the form of three rows and two columns. This application also sends the signal to the Raspberry Pi microcontroller, which is the heart of the device. The controller converts the Braille input control signals received from the mobile application to text file and displays it on the computer. The proposed device has the following four subsystems:

1) A scanner as an input device to scan the text image that is to be read or a downloaded image from the internet or a prevailing image in the system.

2) A Braille display made up of six miniature push pull type solenoids that represent each dot of the Braille alphabet and stroke up to highlight that particular dot.

3) An Android application to take input in Braille language, six movable and connected dots, appears on the screen of the smart phone that serves as an input from the visually disabled.

4) A speaker that gives simultaneous output as produced by the Braille Display.

\section{CONCLUSION}

Despite the increasing attractiveness of voice output for the accessibility of textual information, braille always retrains a preferable position as the medium of communication used by the visually impaired. This paper evaluates the advantages and disadvantages of various Braille display technologies and gives an optimum solution depending on the requisites. Disparate Braille devices are compared on the basis of manifold features involving complexity, reliability and cost. The paper also quick fixes the communication complications while reading and writing text for the blind by proposing a new device plan. Most of the blind people today cannot afford the readily available Braille devices today due to its high cost and also due to importability. Thus the proposed plan emphasizes majorly on cost effectiveness and compactness of the Braille display. Moreover, they do not have to wait for the braille version of a book or journal to come out and can read any normal book. Further upgradation can be made in the outlined device to get the most fitting gadget to aid the unsighted.

Note that the word for Table and Figure are spelled out. Figure's captions should be centered beneath the image or picture, and Table captions should be centered above the table body.

TABLE 1: Comparison of the different types of refreshable Braille displays. (The number of stars represents increasing magnitude.)

\begin{tabular}{|c|c|c|c|}
\hline Braille Display & Complexity & Reliability & Cost \\
\hline Linear & $*$ & $* * *$ & $* *$ \\
\hline Rotating & $* * *$ & $* *$ & $*$ \\
\hline Panda & $* * *$ & Insufficient Data & $* * 4$ \\
\hline
\end{tabular}

\section{ACKNOWLEDGEMENTS}

The authors are overwhelmed by the base provided by the institution, management and the staff. Notwithstanding that any delusion are their own and should not defame the prominence of the esteemed seminary or any persons in routine. Every project big or small is triumphant mostly due to the attempt of a number of remarkable people who have always given their valuable advocacy or lent a helping hand. We earnestly groove on to the inspiration, support and supervision of all those people who have been auxiliary in making it a competent.

\section{REFERENCES}

[1] A. P. Grunwald, "Reading and writing machine using raised Patterns," Nov. 1971.

[2] "The braille literacy crisis in America: Facing the truth, reversing the trend, empowering the blind," Nat. Federation Blind Jernigan Inst., Baltimore, MD, USA, Tech. Rep. 5, 2009.

[3] N. H. Runyan and D. B. Blazie, "The continuing quest for the 'Holy Braille' of tactile displays," in Proc. SPIE 
8107, Nano-Opto- Mech. Syst., Sep. 2011, pp. 81070G1-81070G-17.

[4] M. A. Heller. (2002, Sep.). Tactile picture perception in sighted and blind people. Behav. Brain Res. [Online]. 135(1/2), pp. 65-68. Available: http://www.ncbi.nlm.nih.gov/pubmed/12356435

[5] MahamNadeem, Nida Aziz, Umar Sajjad, Faizan Aziz, Hammad Shaikh, "A comparative analysis of braille generation technologies", 2016 International Conference on Advanced Robotics and Mechatronics (ICARM)
[6] Fung-HueiYeh and Shih-Hao Liang, "Mechanism design of the flapper actuator in Chinese Braille display,"Sensors and Actuators A: Physical, pp. 680-689, 2006.

[7] Y. Hagaa, W. Makishib, K. Iwamib, K. Totsub, K. Nakamurac, and M. Esashid, "Dynamic Braille display using SMA coil actuator and magnetic latch," Sensors and Actuators A: Physical, p. 316-322, 2005

[8] J. Seymour-Ford, History of the Perkins Brailler, Watertown, Massachusetts, 2009

[9] "Mangold L Tape (TM) - Simplified Tactile Display," 7 February 2011. [Online] 